Cite this: Phys. Chem. Chem. Phys., 2014, 16, 6903

Received 5th February 2014

Accepted 26th February 2014

DOI: $10.1039 / c 4 c p 00525 b$

www.rsc.org/pccp

\section{Vortex-induced aggregation in superfluid helium droplets}

\author{
Daniel Spence, Elspeth Latimer, Cheng Feng, Adrian Boatwright, Andrew M. Ellis* \\ and Shengfu Yang*
}

The formation of $\mathrm{Ag}$ nanoparticles by the addition of $\mathrm{Ag}$ atoms to helium droplets has been investigated. The resulting nanoparticles were then imaged by transmission electron microscopy after being deposited on a thin solid surface. In large helium droplets chains of Ag nanorods were observed similar to recently reported track-like deposits [Gomez et al., Phys. Rev. Lett., 2012, 108, 155302]. However, by adjusting the experimental conditions chains of spherical nanoparticles could also be seen with a nearly uniform inter-particle spacing. Given that spherical Ag nanoparticles have no intrinsic anisotropy, the only viable explanation is that these particles must be guided into position by interaction with a quantized vortex spanning the diameter of the helium droplet. Furthermore, addition of Si to the droplets immediately after Ag resulted in Si inserting between the Ag nanoparticles to form continuous nanowires. This eliminates the possibility that the segmented Ag nanostructures are the result of nanowire fragmentation when the helium droplets collide with the deposition substrate. Thus segmented Ag chains are shown to be an intrinsic feature of $\mathrm{Ag}$ aggregation in helium droplets in the presence of a quantized vortex.

\section{Introduction}

Liquid helium is a superfluid at temperatures below $2.17 \mathrm{~K}$. One of the many intriguing properties of the bulk superfluid is that rotational motion manifests itself as fluid circulation around vortex lines with quantized angular momentum. ${ }^{1,2}$ A method for visualizing the very narrow vortices, which have core diameters on the order of an Ångstrom, was developed in 2006. ${ }^{3}$ By bubbling hydrogen highly diluted in helium gas through the liquid helium, condensed hydrogen particles with sizes on the order of microns were produced. Below the superfluid phase transition temperature these particles become pinned to quantized vortices and light scattering then allows images of the vortices to be made. ${ }^{3-6}$ In other recent experiments, laser

Department of Chemistry, University of Leicester, Leicester LE1 7RH, UK. E-mail: sfy1@le.ac.uk, andrew.ellis@le.ac.uk; Fax: +44 (0)116 252 3789; Tel: +44(0)116252 2127 ablation of metals has been used to produce nanowires in bulk liquid helium. ${ }^{7}$ After several hours of continuous ablation very long filaments $(\sim 15 \mathrm{~mm})$ composed of many entangled nanowires with narrow diameters $(2-10 \mathrm{~nm})$ were formed in superfluid helium. In contrast in the non-superfluid liquid (above $2.17 \mathrm{~K}$ ) the nanowires were shorter and showed no aggregation into macroscopic ropes. In view of these observations it seems likely that the formation of large filaments was assisted by the pinning of metal atoms to quantized vortices.

Liquid helium droplets are the nanoscale counterparts of bulk superfluid helium. These droplets can be formed with as few as tens of helium atoms all the way up to micron-sized droplets with well in excess of $10^{9}$ helium atoms. ${ }^{8}$ One of the fascinating opportunities offered by helium droplets is to see how properties identified in bulk superfluid helium transfer into the nanoscale. The behaviour of quantized vortices when restricted to nanoscale dimensions raises the possibility of exploring new physics, as well as new chemistry. However, while well known in the bulk liquid, evidence for quantized vortices in its nanoscale counterpart, superfluid helium droplets, is sparse. Indeed while quantized vortices have been predicted in helium droplets $^{9,10}$ there was no experimental evidence to support this until very recently. The first real indication that quantized vortices may be present in helium droplets came from experiments by Gomez et al., ${ }^{11}$ in which multiple Ag atoms were added to relatively large helium droplets (with diameters $>300 \mathrm{~nm}$ ) and aggregated into nanoparticles. The Ag-doped droplets were then allowed to collide with a substrate and the resulting nanostructures were imaged using transmission electron microscopy (TEM). Track-like deposits consisting of multiple elongated $\mathrm{Ag}$ segments (nanorods) aligned head-to-tail were observed, with the overall lengths of the tracks being broadly consistent with the diameters of the helium droplets. The explanation offered by Gomez et al. for these $1 \mathrm{D}$ nanostructures was that $\mathrm{Ag}$ atoms become pinned to quantized vortices spanning the helium droplets, which directs the formation of elongated structures. However, the observation of aligned nanorods is potentially problematic, since the rods themselves introduce an anisotropy. 
Hence this evidence for quantized vortices in helium droplets is indicative rather than conclusive.

$\mathrm{Ag}$ nanoparticles have been synthesized in superfluid helium droplets and have been investigated using TEM by several research groups. ${ }^{11,12-14}$ Here we investigated the formation of $\mathrm{Ag}$ nanoparticles in large helium droplets in order to further explore the possible role of quantized vortices in the nanoscale superfluid. We report new images supporting the presence of quantized vortices in sub-micron sized helium droplets. In order to test whether the TEM images reflect the real structures of Ag in helium droplets, we added Si to the droplets immediately after the addition of $\mathrm{Ag}$ and see infilling of the gaps between the $\mathrm{Ag}$ nanoparticles. This observation confirms that the separation of $\mathrm{Ag}$ nanoparticles is a feature intrinsic to Ag. We also suggest a possible mechanism to interpret the growth of $\mathrm{Ag}$ nanoparticles in the vicinity of a quantized vortex.

\section{Experimental}

The apparatus for the synthesis of nanoparticles in superfluid helium droplets has been detailed previously. ${ }^{12}$ Briefly, a closedcycle cryostat is used to pre-cool high-purity helium gas (99.9999\%) and helium droplets are then formed by expansion of the helium into vacuum through a pinhole nozzle with an aperture of $5 \mu \mathrm{m}$ diameter. The operating stagnation pressure can be varied from 10-50 bar and the temperature can be accurately controlled from 3 to $30 \mathrm{~K}$. At the lowest temperature helium droplets composed of $\gg 10^{9}$ atoms can be formed by expansion from the liquid phase of the helium behind the pinhole. ${ }^{8,13}$ The cryostat is mounted on an XYZ manipulator, allowing fine control of the position of the nozzle relative to the skimmer.

In this work the stagnation pressure of helium droplets was kept at 15 bar. Ag nanoparticles were formed by addition of $\mathrm{Ag}$ atoms to the helium droplets when they passed through a resistively heated oven containing high-purity Ag. In the case of Ag-Si binary particles we added Si separately in a second oven downstream of the oven used to evaporate Ag. Further downstream is a deposition station coupled with a load-lock chamber, which allows the transportation of deposition targets to external microscopy facilities without interrupting the vacuum for the rest of the apparatus. The nanoparticles were deposited onto lacey carbon substrates, which can be removed from the vacuum for TEM imaging (JEOL JEM-2100 LaB6 transmission electron microscope).

\section{Results}

Our concern here is with evidence for quantized vortices, which seems to be more evident for very large helium droplets. Similar to Gomez et al., we have attempted to observe chains of silver nanoparticles formed in these large helium droplets which might arise from alignment along a quantized vortex.

As well as varying the helium droplet size, a wide range of $\mathrm{Ag}$ vapour pressures have been explored by controlling the oven temperature. We have found that at the lower end, where the
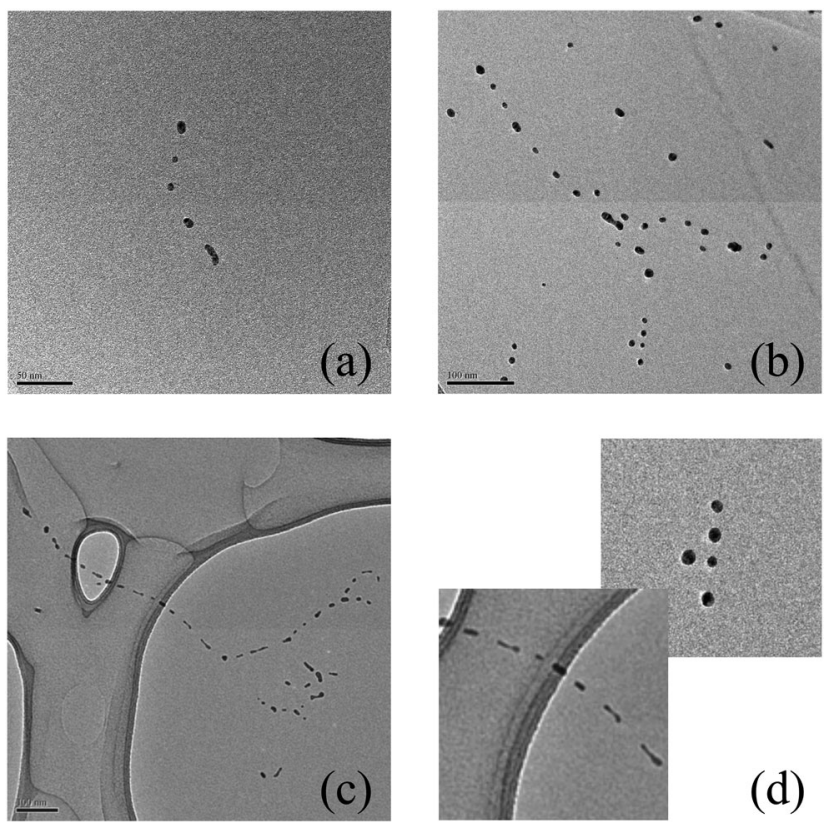

Fig. 1 TEM images of $\mathrm{Ag}$ nanoparticles formed in helium droplets with mean diameters of (a) $790 \mathrm{~nm}$; (b) $1.7 \mu \mathrm{m}$; (c) $3 \mu \mathrm{m}$. The scale marks in the lower left corner of each image are $50 \mathrm{~nm}, 100 \mathrm{~nm}$ and $100 \mathrm{~nm}$, respectively, for (a), (b) and (c). Image (d) shows expanded views of (b) and (c). In image (c) (and the corresponding expanded view) the large scale features are the mesh edges of the lacey carbon substrate.

oven temperature was ca. $1230 \mathrm{~K}$ (slightly lower than the melting point of $\mathrm{Ag}$ ), individual nanoparticles that are largely spherical can be formed. It should also be noted that under low pressure doping conditions the amount of added silver would be insufficient to cover the entire length of a vortex across the helium droplets, but we can nevertheless see one-dimensional structures. Fig. 1(a) shows a chain of Ag nanoparticles derived from helium droplets produced at a nozzle temperature of $5.5 \mathrm{~K}$, with a mean initial droplet diameter estimated as $790 \mathrm{~nm}$ ( $\sim 10^{9}$ helium atoms) according to the recent measurement of helium droplet sizes by Gomez et al. ${ }^{13}$ The diameter of the spheroidal Ag particles is $\sim 6 \mathrm{~nm}$ and the inter-particle spacing is $\sim 22 \mathrm{~nm}$. Fig. 1(b) shows a TEM image for droplets with an initial diameter of about $1.7 \mu \mathrm{m}$ formed at a source temperature of $5 \mathrm{~K}$. The diameters of the spheroidal nanoparticles in Fig. 1(b) are $\sim 10 \mathrm{~nm}$ and the inter-particle spacing is $\sim 16 \mathrm{~nm}$. In the TEM image for the largest droplet size in this study, formed at $4.5 \mathrm{~K}$ ( $3 \mu \mathrm{m}$ or roughly $3 \times 10^{11}$ helium atoms), the particles in the chain change from being roughly spherical to being clearly elongated, as shown in Fig. 1(c). Chains of $\mathrm{Ag}$ nanorods with an overall chain length of $\sim 700 \mathrm{~nm}$ were observed. The individual nanorods in Fig. 1(c) have lengths of $\sim 21 \mathrm{~nm}$, a diameter of $\sim 8 \mathrm{~nm}$, and an average inter-rod spacing of $15 \mathrm{~nm}$. For all three droplet sizes employed here, the separations between the centres of adjacent particles are nearly equal, i.e., $28 \mathrm{~nm}, 26 \mathrm{~nm}$ and $29 \mathrm{~nm}$ for images (a)-(c), respectively.

To verify whether or not the fragmented structure is an intrinsic feature of $\mathrm{Ag}$ nanoparticles in helium droplets, we have also added Si to the helium droplets after first adding Ag, 


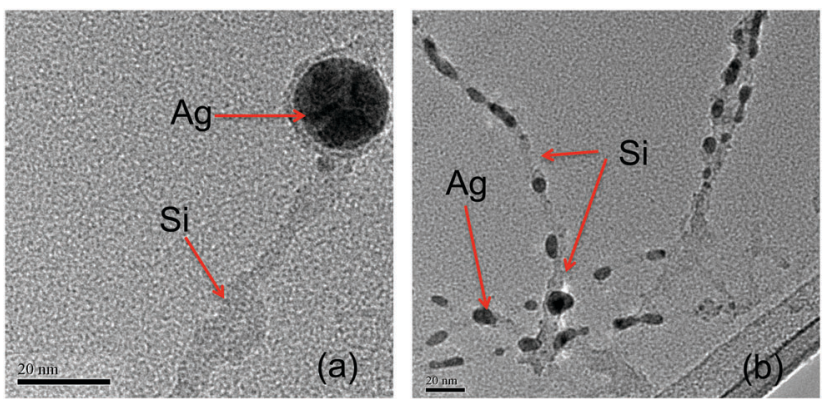

Fig. 2 TEM image of $\mathrm{Ag}-\mathrm{Si}$ nanowires formed by sequential addition of $\mathrm{Ag}$ and Si to helium droplets with an initial diameter of (a) $250 \mathrm{~nm}$ and (b) $3 \mu \mathrm{m}$, respectively. Si provides weaker contrast in TEM than Ag and so the $\mathrm{Si}$ is seen as relatively faint segments.

where the temperature used for the oven that contained Si was $\sim 1400$ K. Fig. 2 shows the consequences of this sequential addition. We started with relatively small helium droplets with a mean droplet diameter of $\sim 250 \mathrm{~nm}$. Under these conditions the distribution of Ag nanoparticles on the deposition substrate is rather random, and we cannot see clear evidence for chains of $\mathrm{Ag}$ nanoparticles on the TEM image. With the addition of $\mathrm{Si}$ a very thin layer of Si was coated on their surface of $\mathrm{Ag}$ nanoparticles, and Si largely grew into nanowires (see Fig. 2(a)). Fig. 2(b) shows the corresponding TEM image for much larger helium droplets, with a diameter of $3 \mu \mathrm{m}$. Here there are continuous chains consisting of separated Ag nanorods but they are linked by continuous Si nanofilaments.

\section{Discussion}

Gomez et al. suggested that one possible explanation for the segmentation of $\mathrm{Ag}$ into nanorods might be the fracture of a continuous $\mathrm{Ag}$ nanowire as the droplet strikes a solid target. ${ }^{11}$ However, our experiments would seem to rule that out. First, the fact that we see chains of spherical particles in the smaller helium droplets indicates that the shape of the deposited nanoparticles is almost unaffected by the landing process. A second and conclusive piece of evidence comes from the addition of Si to the droplet. Remarkably, the Si seems to simply fill in the gaps between the $\mathrm{Ag}$ nanoparticles, creating a continuous chain if sufficient $\mathrm{Si}$ is added. This confirms that the segmentation into $\mathrm{Ag}$ particles in the chains is intrinsic to $\mathrm{Ag}$ rather than all dopants.

The formation of $\mathrm{Ag}$ nanoparticle chains requires an aligning force that acts even when spherical nanoparticles are present in helium droplets, proving beyond doubt that the anisotropy of the nanoparticles is not responsible for chain formation. Instead, the only viable explanation for these chains is that quantized vortices provide the directionality. Quantized vortices can provide an aligning force because atoms and molecules have an increased affinity for the vortices compared with the rest of the superfluid. The attractive force, acting only in the immediate vicinity of the vortex core, is weak and leads to a binding energy of only 3-10 K (2-7 $\left.\mathrm{cm}^{-1}\right)$ for an atom. ${ }^{2}$ However, at the temperature of a helium droplet $\left(0.38 \mathrm{~K}^{15}\right)$ this is strong enough to pin atoms to the vortex.
The fact that both spheroidal nanoparticles and nanorods do not form a continuous nanowire when only $\mathrm{Ag}$ is added to the helium droplets implies that there is a repulsive force in action that prevents aggregation. It is not entirely clear what this repulsive force is but we note that the addition of a particle is expected to distort the vortex core ${ }^{16}$ and when two particles approach substantial vortex curvature is expected, thus raising the energy and providing a repulsive force. Consequently, the inter-particle spacing might be dependent on the balance between the long-range attractive force between adjacent $\mathrm{Ag}$ particles and the repulsive force created by vortex distortion when two particles approach each other. Of course this explanation would imply that $\mathrm{Si}$ is able to overcome this repulsive force and insert between the $\mathrm{Ag}$ spheres/rods. We cannot explain why this happens but clearly $\mathrm{Ag}$ and Si have different interactions with the vortices.

In Fig. 1 we also see a transition from chains of spherical nanoparticles to nanorods when the diameter of the helium droplets increases from $1.7 \mu \mathrm{m}$ to $3 \mu \mathrm{m}$. To provide an explanation we must consider the role of the quantized vortices along with multicentre growth. ${ }^{17,18}$ We can therefore identify three extreme scenarios, which are described below:

(1) In relatively small helium droplets the attractive force between $\mathrm{Ag}$ atoms is overwhelming whether a quantized vortex exists or not. As each $\mathrm{Ag}$ atom is added to the droplet it migrates towards the existing $\mathrm{Ag}_{n}$ cluster because of the reasonably long-range nature of the dispersion interaction and a single spherical nanoparticle forms.

(2) With a relatively low doping rate in much larger helium droplets the odds are likely that a newly added $\mathrm{Ag}$ atom will be too far away from any other atom to be steered together. Thus the Ag atom migrates across the droplet in a semi-random way and may encounter a vortex and attach. If the new $\mathrm{Ag}$ atom approaches a particle already pinned to the vortex aggregation will occur to make a larger particle attached to the vortex. In this way we can envisage new particles being made at different positions along the vortex. Since it is possible for atoms to approach the pinned particles with near equal probability from all directions, the nanoparticles in the chain are expected to be quasi-spherical.

(3) For even larger helium droplets the effective Ag doping rate is so high that the time between successive pickup events is shorter than the migration time for $\mathrm{Ag}$ atoms to reach the vortex line. In this case a number of small Ag nanoparticles can be formed in multiple sites away from any vortex. This is the so-called multicentre growth scenario, which occurs when the migration time of the dopant is much longer than the time between successive pickup events. ${ }^{17,18}$ The multiple particles inside the droplet will eventually migrate and could aggregate with other particles once they become pinned to a vortex, leading to the formation of nanorods. Hence the formation of a chain of nanorods of $\mathrm{Ag}$ in large helium droplets might be the product of the combined action of multi-centre growth and the pinning of particles to a quantized vortex. Furthermore, we cannot rule out the possibility that multiple vortices might form in some of the helium droplets. Vortex arrays are well-known in bulk superfluid helium ${ }^{2}$ and are conceivable in helium droplets if the latter are formed with 
sufficient angular momentum. Multiple vortices would complicate particle aggregation by offering nucleation sites along different vortex lines within a droplet.

We emphasise that the mechanism proposed above is highly speculative and simplistic. Some firm supporting evidence, experimental or theoretical, is needed to establish whether it describes the essential physics of particle aggregation in large helium droplets.

\section{Conclusions}

In conclusion we have observed long chains of $\mathrm{Ag}$ nanoparticles in micron and sub-micron sized superfluid helium droplets and have shown that these segmented structures are formed within the droplets. A key finding is that this one-dimensional growth is seen for both spherical and rod-like nanoparticles, demonstrating that the anisotropy needed to create these long chains comes from the droplet rather than the constituent particles. As far as we can see the only viable explanation is that quantized vortices provide the guiding force to form these elongated objects. The substantial gap between the Ag particles in the chains has been shown to be an intrinsic property of the Ag-vortex interaction, since the gaps can be filled by addition of $\mathrm{Si}$ to the helium droplets.

\section{Acknowledgements}

SY and AME wish to thank the UK Engineering and Physical Sciences Research Council (EPSRC) and the Leverhulme Trust for grants in support of this work. We are grateful to the Advanced Microscopy Centre at the University of Leicester for providing access to TEM facilities.

\section{References}

1 R. P. Feynman, in Progress in Low Temperature Physics, ed. C. J. Gorter, North-Holland, Amsterdam, 1955, vol. 1, p. 1.
2 R. J. Donnelly, Quantized Vortices in Helium II, Cambridge University Press, Cambridge, England, 1991.

3 G. P. Bewley, D. P. Lathrop and K. R. Sreenivasan, Nature, 2006, 441, 588.

4 G. P. Bewley, M. S. Paoletti, K. R. Sreenivasan and D. P. Lathrop, Proc. Natl. Acad. Sci. U. S. A., 2008, 105, 13707.

5 M. S. Paoletti, R. B. Fiorito, K. R. Sreenivasan and D. P. Lathrop, J. Phys. Soc. Jpn., 2008, 77, 111007.

6 E. B. Gordon, A. V. Karabulin, V. I. Matyushenko, V. D. Sizov and I. I. Khodos, Chem. Phys. Lett., 2012, 519-520, 64.

7 V. Lebedev, P. Moroshkin, B. Grobety, E. Gordon and A. Weis, J. Low Temp. Phys., 2011, 165, 166.

8 R. E. Grisenti and J. P. Toennies, Phys. Rev. Lett., 2003, 90, 234501.

9 K. K. Lehmann and R. Schmied, Phys. Rev. B: Condens. Matter Mater. Phys., 2003, 68, 224520.

10 F. Dalfovo, R. Mayol, M. Pi and M. Barranco, Phys. Rev. Lett., 2000, 85, 1028.

11 L. F. Gomez, E. Loginov and A. F. Vilesov, Phys. Rev. Lett., 2012, 108, 155302.

12 A. Boatwright, C. Feng, D. Spence, E. Latimer, C. Binns, A. M. Ellis and S. Yang, Faraday Discuss., 2013, 162, 113.

13 L. F. Gomez, E. Loginov, R. Sliter and A. F. Vilesov, J. Chem. Phys., 2011, 135, 154201.

14 A. Volk, P. Thaler, M. Koch, E. Fisslthaler, W. Grogger and W. E. Ernst, J. Chem. Phys., 2013, 138, 214312.

15 J. P. Toennies and A. F. Vilesov, Angew. Chem., Int. Ed., 2004, 43, 2622.

16 E. B. Gordon, A. V. Karabulin, V. I. Matyushenko, V. D. Sizov and I. I. Khodos, J. Exp. Theor. Phys., 2011, 112, 1061.

17 E. Loginov, L. F. Gomez and A. F. Vilesov, J. Phys. Chem. A, 2011, 115, 7199.

18 E. Loginov, L. F. Gomez, N. Chiang, A. Halder, N. Guggemos, V. V. Kresin and A. F. Vilesov, Phys. Rev. Lett., 2011, 106, 233401. 